\title{
MICROZONIFICACIÓN DEL CULTIVO DE Myrciaria dubia HKB Mc Vaugh "CAMU CAMU” EN SUELOS ALUVIALES DE LA REGIÓN UCAYALI
}

Antonio López ${ }^{1}$, Fernando Rodríguez ${ }^{2}$

\section{RESUMEN}

El estudio tuvo como propósito elaborar una metodología de microzonificación de Myrciaria dubia HBK Mc Vaugh, «camu camu», en base a los requerimientos ambientales del cultivo, en términos de clima, tipo de suelo, pendiente, condiciones del medio, accesibilidad y predisposición de la comunidad por el cultivo. Para ello, primero se procedió a establecer una escala de valoración a cada una de las variables ambientales y posteriormente se seleccionó la zona de trabajo en la localidad de San Francisco, sobre un área total de 100 ha.

El trabajo de campo se realizó en el mes de diciembre del 2000, y para ello se utilizó el método de transectos.

La fase de gabinete se realizó en el Centro de Información de la Amazonía Peruana, en la Unidad de Información Geográfica y Teledetección, del Instituto de Investigaciones de la Amazonía Peruana - IIAP, en el mes de febrero del 2001.

Del trabajo de interpretación de los mapas temáticos de la microzonificación de San Francisco, se han establecido las siguientes conclusiones preliminares: Con relación a la variable textura de suelo, se presenta más de 90 ha de textura buena para el cultivo, con predominancia de las clases arcillosa, arcillo-limosa y franco-arcillolimosa. La textura arcilla franja sólo ocupa cerca de 10 ha.

Respecto a la variable pendiente de terreno, se ha determinado que mayormente existen suelos con buena pendiente, entre 0 y $20 \%$ (53 ha), y pocas áreas ( $11 \mathrm{ha}$ ) con pendiente mayor de $20 \%$, no apta para "camu camu".

1 Investigador del Programa de Ecosistemas Terrestres IIAP Ucayali.

2 Director del Programa de Ordenamiento Ambiental IIAP Iquitos. 
Con relación a la altura de inundación, 33 y 41 ha, muestran muy bueno (menos de 1 $\mathrm{m}$ ) y bueno (entre 1 y $2 \mathrm{~m}$ ) nivel de inundación, respectivamente. $Y$ con respecto a la profundidad efectiva del suelo se ha detectado 39 y 46 ha con niveles bueno (entre 21 y $30 \mathrm{~cm}$ ) y regular (entre 11 a $20 \mathrm{~cm}$ ), respectivamente.

La evaluación de $\mathrm{pH}$ nos indica que la zona de estudio muestra entre 32 y 64 ha con rangos de $\mathrm{pH}$ adecuado (entre 6.1 y 7.3) y moderado (entre 5.1 y 6.0), para el establecimiento del cultivo en esa zona.

Finalmente, recopilando toda la información se ha determinado que existen 86.5 ha con aptitud buena a moderada para la instalación del cultivo en la zona de San Francisco de Asís.

Palabras Clave: Zonificación ecológica, camu camu, suelos aluviales, textura, pendiente, altura de inundación, $\mathrm{pH}$, profundidad efectiva

\section{SUMMARY}

The purpose of this work is to elaborate a methodology for micro zonification of camu camu, Myrciaria dubia HBK Mc Vaugh, based on the environmental requirements of the cultivation, in terms of climate, soil type, slope, stability of the climate, accessibility and willingness of the community for the cultivation. First of all a scale of valuation to each one of the environmental variables was established and later on the work area was selected in the San Francisco town, on a total area of 100 ha. This area is located northeast from Pucallpa on the right riverbank of the Ucayali river (S08 $16^{\prime} 43^{\prime \prime}$ and WO74 $\left.29^{\prime} 24^{\prime \prime}\right)$. Climate corresponds to subtropical humid forest, of plain physiography to lightly bowed with temperatures that oscillate between $19.5^{\circ} \mathrm{C}$ and $31.3^{\circ} \mathrm{C}$, precipitation half monthly variable between 45.2 and $240.3 \mathrm{~mm}$.

Field work was carried in the month of December 2000, using the transect method. First, a base trail of $1000 \mathrm{~m}$ was open on parallel sense to the course of the river. Subsequently, after fixing a first point of the base trail, six (06) strips of $1000 \mathrm{~m}$ of longitude were open each 200 meters in perpendicular form to the base trail. In each strip several sampling points were taken each $50 \mathrm{~m}$ to $100 \mathrm{~m}$, according to how the land physiography.

In each sampling point an evaluation was carried out of each one of the environmental variables, especially those referred to the textural class, effective depth of the floor, slope, and existent vegetation. 
The cabinet phase was carried out in the CIAP-UIGT, in IIAP, in the month of February 2001, through the conditioning of the field information according to the established ranges for each variable in the study.

From the work of interpretation of the thematic maps of San Francisco's micro zonification the following preliminary conclusions have settled down:

With regard to the floor texture variable, more than 90 ha were found with good texture for cultivation, with predominance of the loamy, arcillo-oozy and franc-arcillooozy classes. The clay fringe texture only squatters near 10 has

Regarding the pending variable of land it has been determined that mostly floors exist with good slope between 0 and 20\% (53 ha) and few areas (11 ha) with slope bigger than $20 \%$ ) not capable for camu camu

With respect to the flood height, 33 and 41 ha show a very good (less than $1 \mathrm{~m}$ ) and good (between 1 and $2 \mathrm{~m}$ ) flood level, respectively. And with regard to the effective depth of the floor 39 and 46 ha have been detected with good levels (between 21 and $30 \mathrm{~cm}$ ) and regulate levels (among 11 to $20 \mathrm{~cm}$ ), respectively.

The $\mathrm{pH}$ evaluation indicates us that the study area shows between 32 and 64 ha with ranges of appropriate $\mathrm{pH}$ (between 6.1 and 7.3) and moderate (among 5.1 at 6.0) for the establishment of cultivation in that area.

Finally, in gathering all the information it has been determined that there exist 86.5 ha with good to moderate aptitude for the installation of cultivation in San Francisco.

Key words: Ecological zonification, camu camu, alluvial soils, texture, slope, flood height, $\mathrm{pH}$, effective depth

\section{INTRODUCCIÓN}

En la Amazonía peruana, los suelos aluviales se encuentran localizados principalmente en la selva baja, en ambos márgenes de los ríos Amazonas, Marañón y Ucayali. Según estudios de la ONERN (1982), estas tierras comprenden una superficie total de 3278500 ha, de las cuales 655400 serían tierras aptas para cultivos en limpio.

Desde este punto de vista, los suelos de áreas inundables constituyen un recurso de gran importancia para el desarrollo de la selva baja, principalmente de los departamentos de Loreto y Ucayali, pues, se estima que en estas tierras se desarrolla 
cerca del $80 \%$ de la actividad agrícola y se localiza el 90\% de los pequeños centros poblados del área rural (Rodríguez, et al., 1985).

En un estudio de zonificación agro ecológica realizado por el IIAP, en 1996, se encontró que dentro de 9 zonas agro-ecológicas, la zona 3 que abarca el área actual de trabajo, se encuentra distribuida en áreas inundables. Fisiográficamente son terrazas bajas originadas por el río Ucayali, su relieve es plano ( 0 a $2 \%$ de pendiente) con una profundidad efectiva profunda. Poseen textura media, con bajo contenido de materia orgánica, alta saturación de bases, $\mathrm{pH}$ neutro y contenido medio de fósforo. El uso actual de estas tierras está relacionado a cultivos temporales y algunas frutas como mango, caimito, huito, mamey y taperibá, entre otros.

En este marco, y con el propósito de orientar las acciones de promoción del cultivo de "camu camu", el IIAP, en 1997, inició la siembra de 800 ha en las márgenes del río Ucayali. Sin embargo, era necesario definir una metodología para determinar las áreas más apropiadas en base al contraste de la demanda ambiental del cultivo de "camu camu" con la oferta ambiental del medio biofísico y socioeconómico.

Como la oferta ambiental, incluye el conocimiento de un significativo número de variables, y estudiarla en toda su dimensión requiere grandes recursos y tiempo, en el presente trabajo, la metodología de microzonificación sólo considera el estudio de aquellas variables que son determinantes para la implantación, desarrollo y producción del «camu camu».

\section{METODOLOGÍA DESARROLLADA}

El proceso metodológico definido para la microzonificación del cultivo de "camu camu" en suelos aluviales involucró los siguientes pasos:

\section{$1^{0}$ Etapa preliminar de gabinete}

Esta fase consistió en elaborar una metodología de microzonificación de "camu camu", en base a la demanda de los requerimientos ambientales del cultivo, en términos de clima, tipo de suelo, pendiente, estabilidad del medio, accesibilidad y predisposición de la comunidad por el cultivo, y se procedió a establecer una escala de valoración para la elaboración de las unidades ecológicas.

Luego, se definió la zona de trabajo en la localidad de San Francisco, sobre un área total de 100 ha. Para esta labor, primero se usó información cartográfica del Instituto 
Geográfico Nacional, y de las imágenes satélite que adquirió el IIAP para desarrollar la zonificación de la cuenca del Aguaytía, a fin de establecer la ubicación geográfica.

La localidad de San Francisco se encuentra ubicada al noreste de Pucallpa a la margen derecha del río Ucayali, entre las coordenadas $\mathrm{S} 08^{\circ} 16^{\prime} 43^{\prime \prime}$ y WO $74^{\circ} 29^{\prime 2} 24^{\prime \prime}$. Su clima corresponde al de bosque húmedo subtropical, de fisiografía plana a ligeramente inclinada, con temperaturas que oscilan entre $19.5^{\circ} \mathrm{C}$ y $31.3^{\circ} \mathrm{C}$, precipitación media mensual variable entre 45.2 y $240.3 \mathrm{~mm}$.

A través del Programa de Agroexportación de «camu camu» se instaló, entre 1997 y 1999, cerca de 80 ha de esta especie a lo largo de esta cuenca, aunque sólo un escaso porcentaje de estas tierras tienen iniciado su fase de producción

\section{$2^{\circ}$ Fase de campo}

El trabajo de campo se realizó en el mes de diciembre del 2000, y para ello se utilizó el método de transectos. Por este método, primero se hizo un recorrido por todo el campo y luego de fijar el punto inicial (sobre la base de GPS), se procedió a abrir una trocha base de $1000 \mathrm{~m}$ en sentido paralelo al curso del río. Seguidamente, partiendo del primer punto de la trocha base, se procedió a abrir seis (6) fajas de $1000 \mathrm{~m}$ de longitud, cada $200 \mathrm{~m}$, en forma perpendicular a la trocha base. En cada faja se tomó varios puntos de muestreo cada 50 a $100 \mathrm{~m}$, según como se presentaba la fisiografía del terreno.

En cada punto de muestreo se realizó una evaluación de cada una de las variables ambientales, especialmente las referidas a la clase textural, profundidad efectiva del suelo, pendiente, y vegetación existente. No se pudo construir una calicata de $1.50 \mathrm{~m}$ por la intensidad de las lluvias en esta época.

Se registraron también, las ocurrencias fisiográficas, como por ejemplo: si cruza una quebrada o caño, existencia de cultivos o purmas, de uno a otro punto, de modo que esta información nos permita establecer finalmente un mapa de uso de la tierra.

\section{$3^{\circ}$ Etapa final de gabinete}

Esta fase se realizó en el CIAP-UIGT, en el mes de febrero del 2001, a través de la siguiente metodología:

1. Acondicionamiento de la información de campo de acuerdo a los rangos establecidos para cada variable en estudio: 


\begin{tabular}{|c|c|c|}
\hline Variable & Escala & Rangos \\
\hline \multicolumn{3}{|l|}{ Pendiente } \\
\hline Buena & 1 & $0-10 \%$ \\
\hline Regular & 2 & $11-20 \%$ \\
\hline Mala & 3 & $21-30 \%$ \\
\hline \multicolumn{3}{|l|}{ Textura } \\
\hline Buena & 1 & $\mathrm{Ar} / \mathrm{ArLo} / \mathrm{FrArLo}$ \\
\hline Regular & 2 & $\mathrm{ArFr}$ \\
\hline Mala & 3 & Ao \\
\hline \multicolumn{3}{|l|}{$\mathrm{PH}$} \\
\hline Bueno & 1 & $6.1-7.3$ \\
\hline Regular & 2 & $5.1-6.0$ \\
\hline Malo & 3 & menor a 5.1 \\
\hline \multicolumn{3}{|c|}{ Inundación } \\
\hline Buena & 1 & $0-100 \mathrm{~cm}$ \\
\hline Regular & 2 & $100-200 \mathrm{~cm}$ \\
\hline Mala & 3 & mayor de $200 \mathrm{~cm}$ \\
\hline \multicolumn{3}{|c|}{ Profundidad efectiva del suelo } \\
\hline Buena & 1 & $21-30 \mathrm{~cm}$ \\
\hline Regular & 2 & $11-20 \mathrm{~cm}$ \\
\hline Mala & 3 & $0-10 \mathrm{~cm}$ \\
\hline
\end{tabular}

2. Digitalización de datos: automatización de la información en CIAP-UIGT.

3. Edición de la información para conectar las áreas no conectadas, borrar las demás y generar la topología (establecer las relaciones espaciales entre los elementos del mapa).

4. Superposición de las 5 coberturas $(\mathrm{pH}$, pendiente, textura, nivel de inundación, profundidad efectiva del suelo).

5. Calificación de áreas por microzonificación en base a los criterios seleccionados en la base de datos.

6. Confección del mapa base a escala 1:5 000, en programa ArcView.

7. Ploteo (impresión) de los mapas temáticos y de microzonificación. 


\section{RESULTADOS Y DISCUSIÓN}

\subsection{Del trabajo preliminar de gabinete}

Con la participación de especialistas del cultivo de "camu camu", se estructuró la metodología de microzonificación de este cultivo en suelos aluviales, que incluye el análisis de variables ambientales determinantes para la instalación y producción del cultivo en suelos aluviales. Estas variables son:

Fisiografía: En zonas aluviales, son recomendables para "camu camu", las restingas bajas con buen drenaje, cuya duración de la inundación no sea mayor a los 4 meses.

En este sentido se sugiere la siguiente escala:

1. Barrizales no aptos para "camu camu"

2. Restingas bajas a medias con buen drenaje: apta para "camu camu"

3. Restingas bajas a medias con mal drenaje: no apta para "camu camu"

4. Restingas altas: no aptas para "camu camu"

Textura de la capa superficial del suelo: En estos suelos jóvenes, el nivel de fertilidad está relacionado con el tamaño de las partículas inorgánicas, en tal sentido, las áreas más propicias son aquellas con textura fina, mientras que las menos propicias son las arenosas. Para el efecto, se propone la siguiente escala:

1. Clase franco-arcillo-limosa apta

2. Clase franco-limosa apta

3. Clase franco-arenosa no apta

4. Clase franco arcillosa no apta

5. Clase arenosa no apta

Profundidad efectiva del suelo: El "camu camu" requiere de un espacio radicular adecuado para captar sin problemas los nutrientes de la capa superficial del suelo. Por consiguiente, las áreas más adecuadas serán aquellas que posean mayor espesor de esta capa superficial.

Por tanto, una escala a usar para este factor puede ser:

1. De 0 a $20 \mathrm{~cm} \quad$ No apta

2. De 20 a $30 \mathrm{~cm}$ Moderadamente apta

3. Mayor de $30 \mathrm{~cm}$ Adecuada para "camu camu" 
Distancia de las parcelas con relación al río: Se sugiere aplicar la siguiente escala:

1. Distancia de 0 a $500 \mathrm{~m}$

2. Distancia de 500 a $1000 \mathrm{~m}$

3. Distancia mayores a $1000 \mathrm{~m}$
No recomendable

Recomendable

No recomendable

- Circulación del agua: Está relacionada con el nivel de oxigenación de la planta, en base a la duración de la inundación. Aguas estancadas durante un buen tiempo, repercuten o afectan en el crecimiento normal de la planta.

Por tanto, una escala para esta variable puede ser:

1. Agua circulante Buena para "camu camu"

2. Agua estancada No adecuada para "camu camu"

Altura de inundación del suelo:

1. Hasta $1 \mathrm{~m}$ Muy bueno

2. Entre 1 a $2 \mathrm{~m}$ Bueno

3. Mayor de $2 \mathrm{~m}$ Malo

\section{Procesos de sedimentación}

Sitios con una dinámica muy fuerte de sedimentación de partículas gruesas (arenosas), por estar ubicadas en ambientes hidrológicos muy dinámicos (fuertes corrientes), son las menos propicias para el cultivo, por cuanto la sedimentación puede tener doble efecto, la primera que si después de una inundación se registra depósitos de sedimentos ( ejemplo, 1 metro o más) causa la muerte de la planta por efecto físico, o cuando el depósito de arena cambia las características superficiales de la textura del suelo (muerte química por falta de nutrientes). De igual forma, la sedimentación de arena es un factor crítico para el sistema de cultivos anuales que acompañan al «camu camu» en los primeros años de su instalación. En este sentido, para el caso de la sedimentación, se propone la siguiente escala:

1. Con sedimentación de arena

No apta

2. Sin sedimentación de arena

Apta

Por otro lado, se ha establecido el mapa de ubicación geográfica de la zona de trabajo, mediante el uso del software Arc View, en un área de 100 ha. 


\subsection{Del trabajo final de gabinete}

A partir del trabajo de interpretación de los mapas temáticos de la microzonificación de San Francisco, se han establecido las siguientes conclusiones preliminares:

Con relación a la variable textura de suelo, se presenta más de 90 ha de textura buena para el cultivo, con predominancia de las clases arcillosa, arcillo-limosa (ArLo) y franco-arcillo-limosa (FrArLo). La textura arcilla franca (ArFr) sólo ocupa cerca de 10 ha.

Respecto a la variable pendiente de terreno, se ha determinado que mayormente existe suelos con buena pendiente entre 0 y $20 \%$ (53 ha) y pocas áreas (11 ha) con pendiente mayor de $20 \%$, no apta para «camu camu».

Con relación a la altura de inundación, 33 y 41 ha muestran, respectivamente, bueno (menos de $1 \mathrm{~m}$ ) y regular (entre 1 y $2 \mathrm{~m}$ ) nivel de inundación. Y con respecto a la profundidad efectiva del suelo, se ha detectado 39 y 46 ha con niveles bueno (entre 21 y $30 \mathrm{~cm}$ ) y regular (entre 11 a $20 \mathrm{~cm}$ ), respectivamente.

La evaluación de $\mathrm{pH}$ nos indica que la zona de estudio muestra entre 32 y 64 ha con rangos de $\mathrm{pH}$ adecuado (entre 6 y 7.3 ) y moderado (de 5.1 a 6.0) para el establecimiento del cultivo en esa zona.

Finalmente, recopilando toda la información se ha determinado que existe 86.5 ha con aptitud buena a moderada para la instalación del cultivo de «camu camu» en la zona de San Francisco de Asís.

En anexos se presentan los mapas temáticos de las variables textura de suelo, pendiente, $\mathrm{pH}$, nivel de inundación y profundidad efectiva, así como el mapa final de micro zonificación de «camu camu», que recoge las distintas combinaciones de mapas temáticos, de acuerdo a la metodología desarrollada en el CIAP-UIGT del IIAP. 


\section{BIBLIOGRAFÍA CONSULTADA}

IIAP COMITÉ REFORESTACION PUCALLPA. 1996. Zonificación agroecológica de la zona aluvial del río Ucayali, entre la boca del río Pachitea y Tiruntan. Iquitos. $80 \mathrm{p}$.

IIAP CTAR Ucayali.2000. Zonificación ecológica económica de la cuenca del río Aguaytía. Informe final. Marzo 2000. Iquitos. 4 volúmenes.

MINISTERIO DE RELACIONES EXTERIORES. 1998. Manual de zonificación ecológica-económica para la Amazonía peruana. Convenio TCA-BID. Programa de Apoyo a la Zonificación de la Amazonía. Lima. 153 p.

IIAP 2000. Manual del camu camu en restingas. Iquitos.( por publicar)

RIVA. R. 1997. Tecnología del cultivo de camu camu Myrciaria dubia HBK Mc Vaugh. en la Amazonía Peruana. INIA-CTAR Ucayali.Pucallpa. 45 p.

VILLACHICA H. 1998. El cultivo de camu camu Myrciaria dubia HBK Mc Vaugh. en la Amazonía Peruana. Tratado de Cooperación Amazónica. Lima. 94 p.

\section{AGRADECIMIENTOS}

Se agradece la colaboración de la comunidad de San Francisco de Asís, por el apoyo en el trabajo de campo; a Rita Riva, Mario Pinedo, José Sanjurjo, del IIAP, por la revisión y composición de mapas en el CIAP-UIGT; así como a los doctores Risto Kaliolla y Óscar Loli por sus comentarios al artículo. 


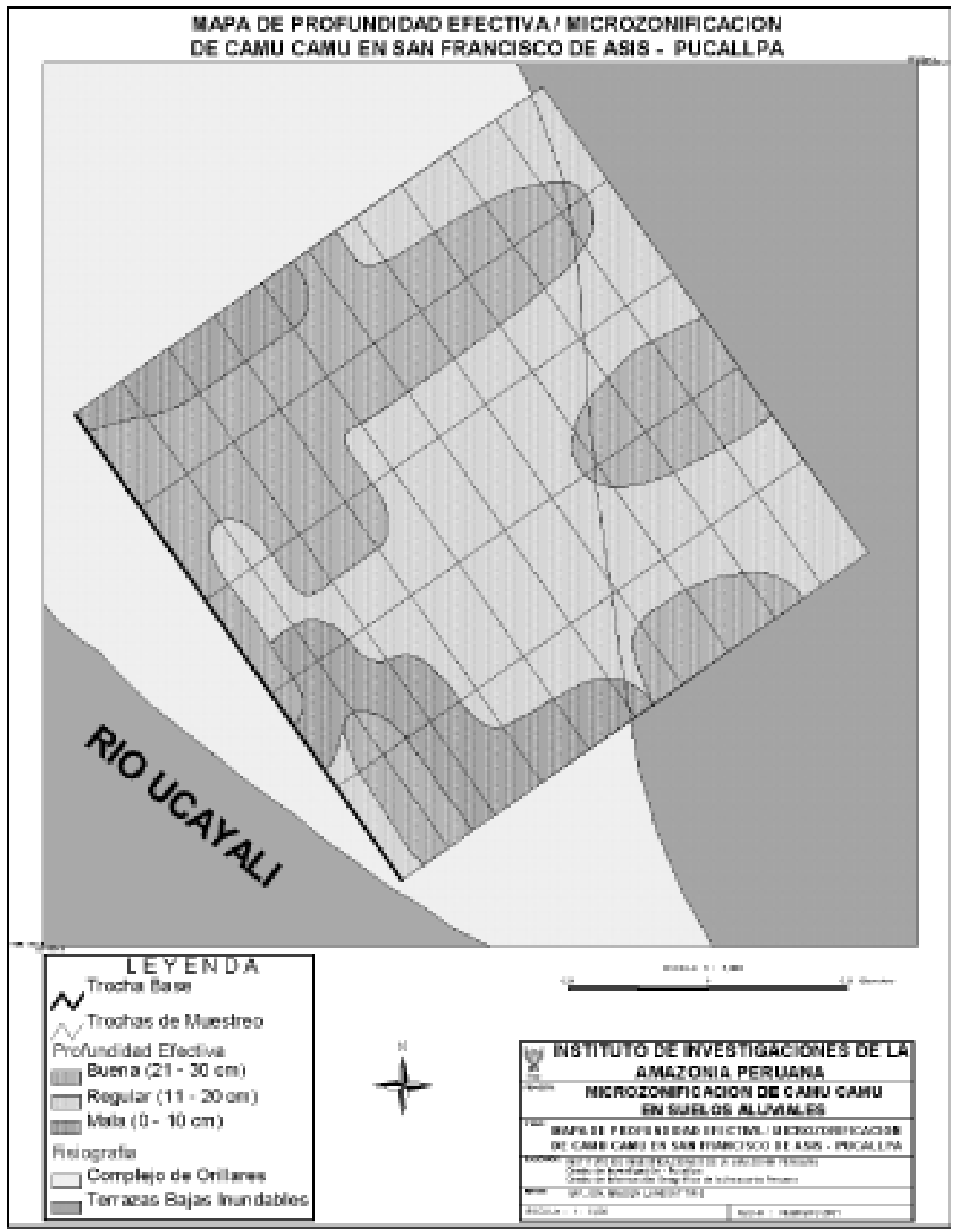




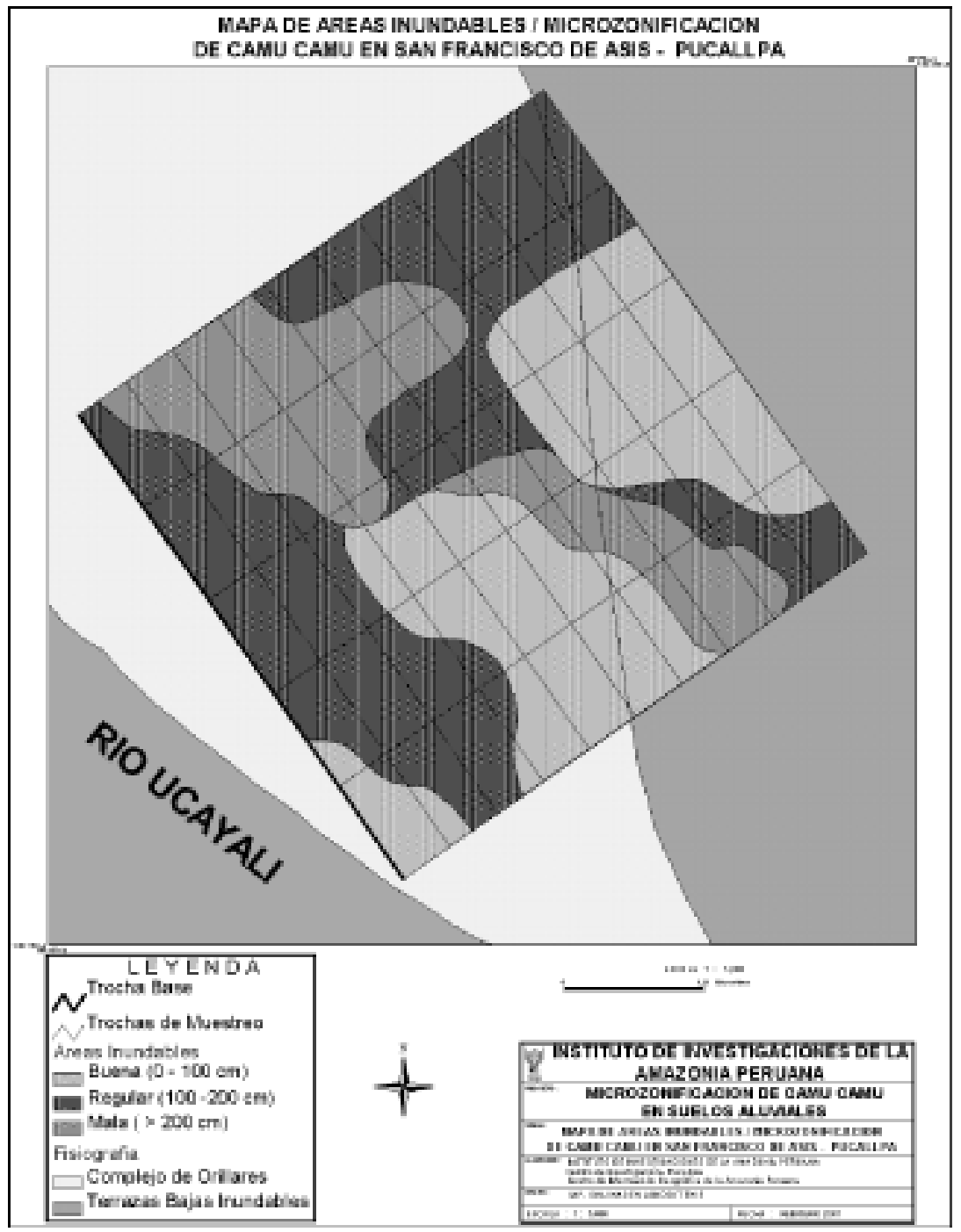




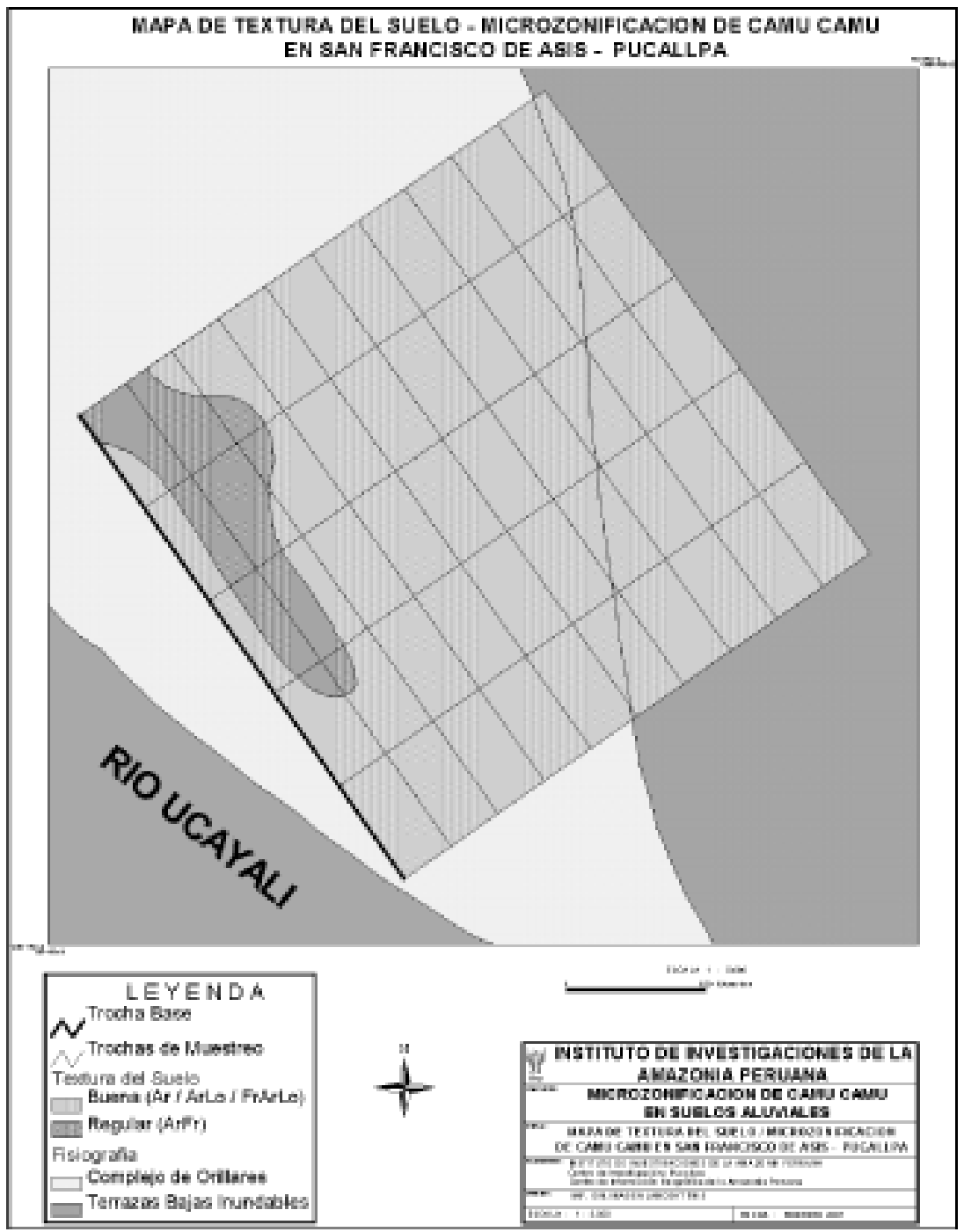




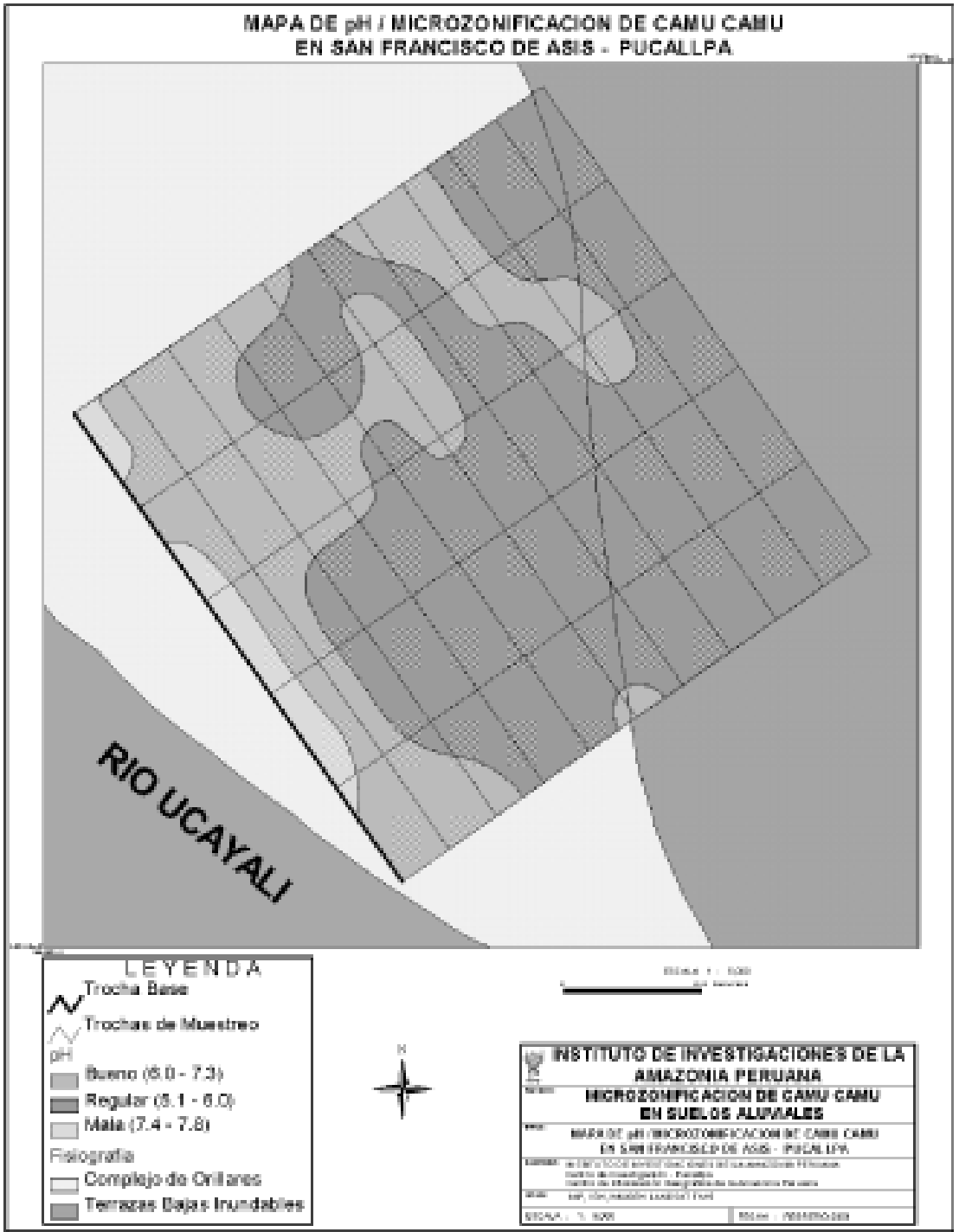




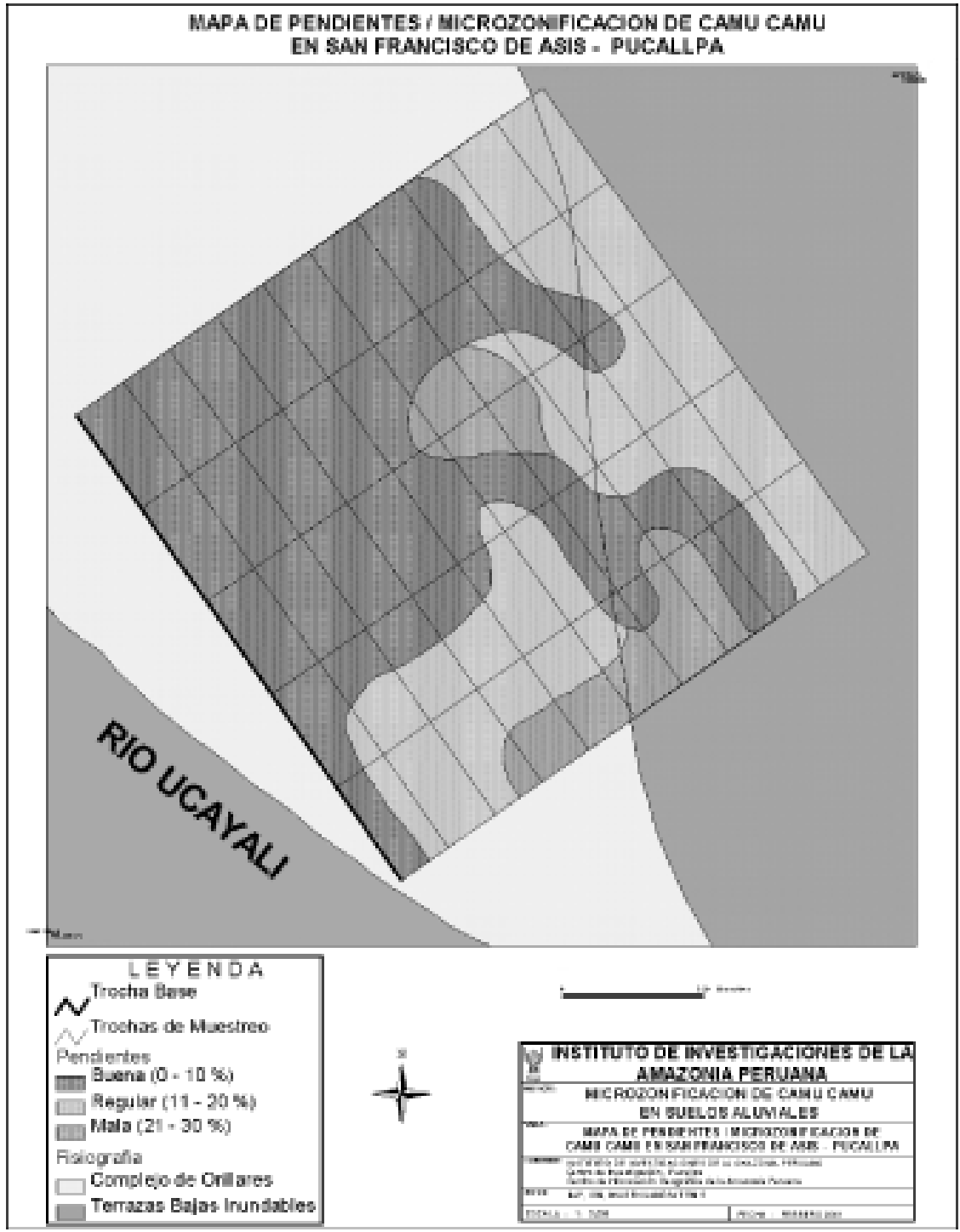




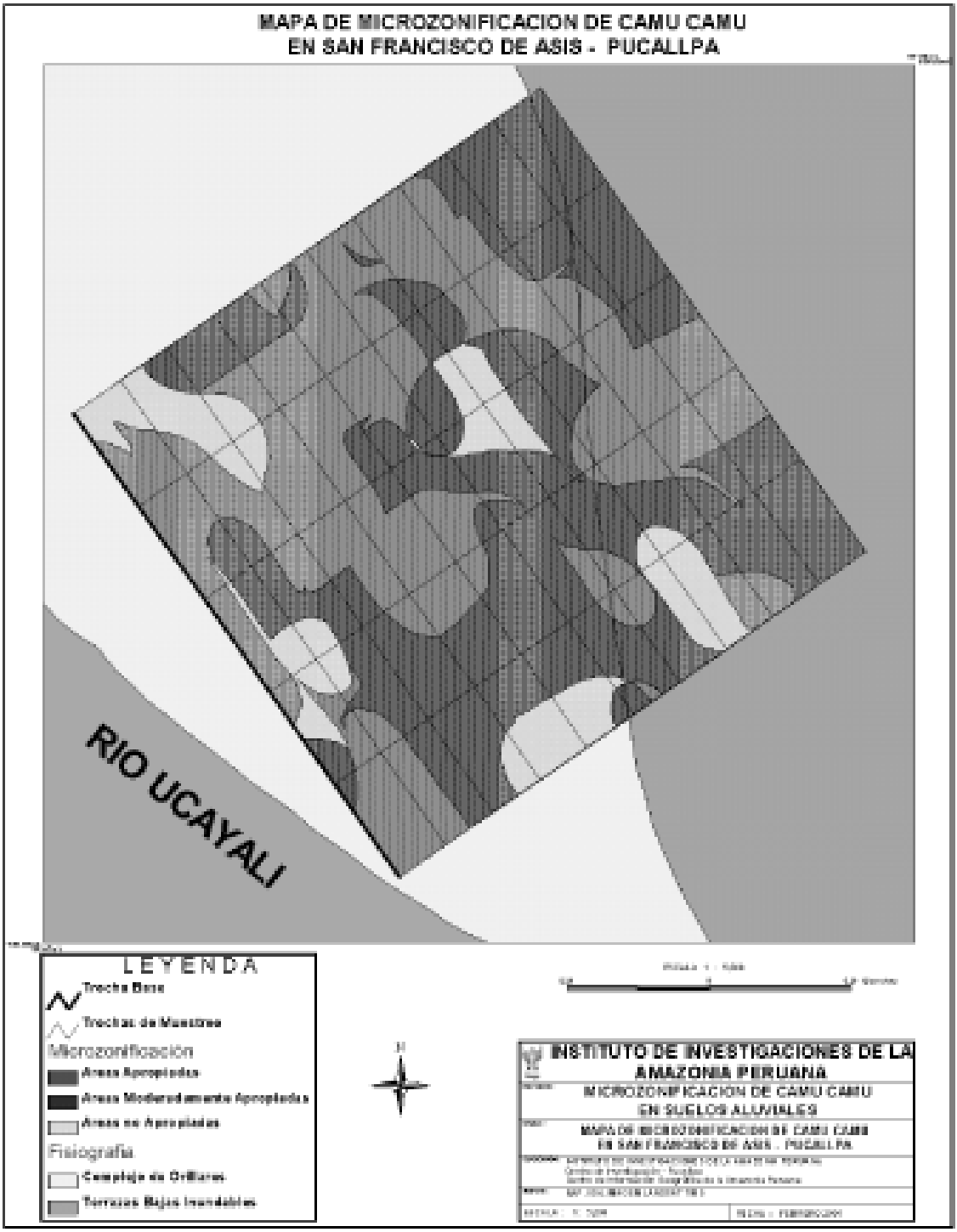

